\title{
Ceftriaxone-Induced Pancreatitis
}

\author{
Muneerah M Albugami, Mohamed Ahmed, and Abdulelah Bin Shihah
}

Can J Hosp Pharm. 2021;74(3):291-3

DOI: 10.4212/cjhp.v74i3.3157

\section{INTRODUCTION}

Ceftriaxone, a widely used third-generation cephalosporin, has been implicated in causing acute pancreatitis. ${ }^{1}$ Risk factors for ceftriaxone-induced acute pancreatitis include biliary stasis, renal failure, and fluid restriction. ${ }^{2,3}$ Ceftriaxoneinduced acute pancreatitis can be managed conservatively, but in some cases more invasive interventions, such as endoscopic retrograde cholangiopancreatography (ERCP), may be required. ${ }^{3-7}$ We describe a 70 -year-old man with ceftriaxoneinduced acute pancreatitis, which was treated with conservative therapy.

\section{CASE REPORT}

A 70-year-old man presented with undocumented fever and cough of several days' duration. ${ }^{*}$ His history was significant for nasopharyngeal carcinoma, which was in remission, adrenal insufficiency, hypothyroidism, and vocal cord paralysis. His home medications consisted of levothyroxine $75 \mu \mathrm{g}$ daily, omeprazole $20 \mathrm{mg}$ once daily, cholecalciferol 1000 units daily, calcium carbonate $600 \mathrm{mg}$ twice daily, and hydrocortisone orally $15 \mathrm{mg}$ in the morning and $5 \mathrm{mg}$ in the evening.

Upon admission, the patient was afebrile and hypoxic, with blood pressure of $97 / 65 \mathrm{~mm} \mathrm{Hg}$, heart rate of $86 / \mathrm{min}$, and respiratory rate of $22 / \mathrm{min}$. He was underweight, with a body mass index of $18.2 \mathrm{~kg} / \mathrm{m}^{2}$. Laboratory tests on admission revealed white blood cells $15.5 \times 10^{9} / \mathrm{L}$ (normal range 3.6-9.6 × 109/L), hemoglobin $132 \mathrm{~g} / \mathrm{L}$ (normal range 129$169 \mathrm{~g} / \mathrm{L}$ ), and platelets $156 \times 10^{9} / \mathrm{L}$ (normal range $140-380 \times$ $10^{9} / \mathrm{L}$. His hepatic profile was normal. He had acute kidney injury secondary to dehydration, with a serum creatinine of $136 \mu \mathrm{mol} / \mathrm{L}$ and creatinine clearance of $30 \mathrm{~mL} / \mathrm{min}$.

Radiography and computed tomography (CT) of the chest showed evidence of bilateral infiltration. Communityacquired pneumonia or aspiration pneumonia was diagnosed. The results of respiratory viral screening and culture of sputum and blood were all negative. Antibiotic therapy was started, consisting of ceftriaxone $2 \mathrm{~g}$ IV daily for 14 days and azithromycin $500 \mathrm{mg}$ orally once daily for 3 days. In addition, IV fluid therapy, in the form of sodium chloride $0.9 \%$, was started at a rate of $100 \mathrm{~mL} / \mathrm{h}$. The patient's home medications were continued at the same doses, with the exception of hydrocortisone, for which the dose was doubled because of the infection.

On day 14 of the admission, the patient reported sudden onset of dyspepsia, nausea, vomiting, epigastric pain, and fever. He denied dysuria, change in the colour of urine or stools, or change in bowel habits. On examination, he was thin with a nontoxic appearance; he was afebrile and tachycardic, with blood pressure of 110/61 mm Hg. His abdomen was tender all over, most notable in the epigastric area, but otherwise soft and lax, with no organomegaly. There were no signs of jaundice, and the findings for other aspects of the physical examination were unremarkable.

The patient's past medical history was negative for liver disease and hemolysis. Additionally, there was no family or personal history of gallstones, and the patient denied any history of alcohol consumption or smoking. The patient had never undergone ERCP, nor was there recent surgical history that might have pointed to the cause of pancreatitis. The laboratory results showed a significant increase in transaminases (aminotransferases), amylase, and lipase levels, relative to values on day 1 of the admission (Table 1).

Ultrasonography of the abdomen revealed mild thickening of the gallbladder wall, with no hyperemia, pericholecystic fluid, or gallstones. The patient was treated conservatively, with IV fluids in the form of sodium chloride $0.9 \%$; in addition, the ceftriaxone was discontinued. Within 48 hours (day 16 of the admission), serum lipase levels and hepatic function improved significantly, with rapid resolution of his symptoms.

A diagnosis of ceftriaxone-induced acute biliary pancreatitis was made. Elevation of liver enzymes in the setting of acute pancreatitis was suggestive of ceftriaxone-associated biliary pseudolithiasis. We could not find any other factors that might have explained the acute pancreatitis. The Naranjo probability scale ${ }^{8}$ indicated a probable relationship (Naranjo score of 7) between the ceftriaxone therapy and the adverse effect of acute pancreatitis in this patient. The patient recovered and was discharged in a stable condition.

*The patient provided consent for publication of this report. 
TABLE 1. Laboratory Data during Hospital Admission

\begin{tabular}{|c|c|c|c|c|}
\hline Laboratory Value & Normal Range & Day 1 & Day 14 & Day 16 \\
\hline \multicolumn{5}{|l|}{ Renal profile } \\
\hline Creatinine $(\mu \mathrm{mol} / \mathrm{L})$ & $64-115$ & 136 & 86 & 81 \\
\hline Urea (mmol/L) & $2.5-7.5$ & 9.6 & 5.5 & 5.7 \\
\hline \multicolumn{5}{|l|}{ Hepatic profile and enzymes } \\
\hline Alanine aminotransferase (U/L) & $10-45$ & 10 & 285.7 & 131 \\
\hline Aspartate aminotransferase (U/L) & $10-45$ & 20.1 & 211.1 & 48.8 \\
\hline Alkaline phosphatase (U/L) & $50-116$ & 84 & 246 & 174 \\
\hline Amylase (U/L) & $30-110$ & - & 3250 & - \\
\hline Lipase (U/L) & $0-60$ & - & 1644 & 67 \\
\hline Triglycerides (mmol/L) & $<1.7$ & - & 1.05 & 0.77 \\
\hline
\end{tabular}

\section{DISCUSSION}

Acute pancreatitis is an acute inflammatory condition of the pancreas. Gallstones and alcohol are the most common causes, accounting for the majority of cases in adult patients. ${ }^{9}$ Medications have also been recognized as a possible cause. ${ }^{10}$ Reports of drug-induced acute pancreatitis range from $0.1 \%$ to $2 \%$ of overall cases. ${ }^{10-12}$ There is no specific test for establishing a diagnosis of drug-induced acute pancreatitis; instead, the diagnosis is often based on exclusion of all other common causes of acute pancreatitis.

Globally, few cases of ceftriaxone-associated pancreatitis have been reported. ${ }^{1,4-7,13,14}$ Ceftriaxone is primarily excreted through the kidneys, and $10 \%$ to $20 \%$ of the drug is excreted in the bile. ${ }^{13}$ Precipitation of ceftriaxone in the bile causes the formation of biliary sludge, leading to the development of cholangitis, cholecystitis, or acute pancreatitis. ${ }^{13}$ Ceftriaxone harbours high calcium-binding affinity, and the solubility of the calcium-ceftriaxone complex is low; as such, the bound substance tends to be retained in the bile. ${ }^{12}$ Ceftriaxone forms a precipitate after excretion and concentration in bile in the gallbladder, the major constituent of which is a calcium-ceftriaxone salt. ${ }^{4}$

Pseudolithiasis is a term used for imaging abnormalities observed in the gallbladder or common bile duct in patients treated with ceftriaxone, to differentiate these ceftriaxoneinduced reversible abnormalities from those related to truly operable stones. ${ }^{1}$ It is difficult to differentiate ceftriaxoneassociated sludge from the usual gallbladder sludge on ultrasonographic or CT images, and the pseudolithiasis can rapidly disappear after discontinuation of ceftriaxone. ${ }^{4}$ Long-term IV administration of high-dose ceftriaxone has been associated with the transient formation of biliary sludge, which is usually reversible upon discontinuation of the drug. ${ }^{1}$

Review of the existing case reports of ceftriaxoneinduced acute pancreatitis revealed that it can occur in both men and women of any age. The duration of treatment with ceftriaxone in these cases varied from 2 days to several weeks, with typical adult doses of 2 to $4 \mathrm{~g} /$ day. Management of ceftriaxone-induced acute pancreatitis was variable and included conservative treatment, ERCP, or cholecystectomy. ${ }^{1,4-7,13,14}$ Risk factors for acute pancreatitis associated with ceftriaxone include poor oral intake, hypoalbuminemia, and renal impairment, which can increase the incidence of pseudolithiasis. This is because about $90 \%$ of ceftriaxone is bound to serum albumin and about $55 \%$ of the drug is excreted by the kidney. ${ }^{2,3}$ In the case reported here, the patient's poor oral intake and renal impairment put him at risk of acute pancreatitis. Renal dysfunction has been identified as an independent risk factor for ceftriaxone-associated acute pancreatitis in adults. ${ }^{15}$ When ceftriaxone is administered to patients who have renal insufficiency or are receiving dialysis, it may be necessary to adjust the dosage because high concentrations of ceftriaxone in the blood and bile may cause biliary pseudolithiasis. ${ }^{16}$ Additionally, macrolides have been associated with acute pancreatitis. Most reports have identified erythromycin as a leading cause, but other macrolides, such as roxitromycin and clarithromycin, have also been associated with acute pancreatitis. ${ }^{16}$ The mechanism by which erythromycin can cause this adverse effect may be its prokinetic activity, which can lead to spasm of the sphincter of Oddi. The fact that our patient was being treated with azithromycin may have increased his risk of acute pancreatitis.

\section{References}

1. Zimmermann AE, Katona BG, Jodhka JS, Williams RB. Ceftriaxoneinduced acute pancreatitis. Ann Pharmacother. 1993;27:36-7.

2. Imafuku A, Sawa N, Sekine A, Kawada M, Hiramatsu R. Risk factors of ceftriaxone-associated biliary pseudolithiasis in adults: influence of renal dysfunction. Clin Exp Nephrol. 2018;22(3):613-9.

3. Murata S, Aomatsu T, Yoden A, Tamai H. Fasting and bed rest, even for a relatively short period, are risk factors for ceftriaxone-associated pseudolithiasis. Pediatr Int. 2015;57(5):942-6.

4. Maranan MC, Gerber SI, Miller GG. Gallstone pancreatitis caused by ceftriaxone. Pediatr Infect Dis J. 1998;17(7):662-3.

5. Famularo G, Polchi S, De Simone C. Acute cholecystitis and pancreatitis in a patient with biliary sludge associated with the use of ceftriaxone: a rare but potentially severe complication. Ann Ital Med Int. 1999;14(3): 202-4.

6. Sasaki Y, Aoki S, Aoki K, Achiwa K, Yama T, Kubota M, et al. [Acute pancreatitis associated with the administration of ceftriaxone in an 
adult patient]. Nihon Shokakibyo Gakkai Zasshi. 2009;106(4):569-75. Article in Japanese.

7. Ruggiero S, Di Nardo R, Polimeno T, Rossi F, Capuano A. Ceftriaxone induced pancreatitis in a pediatric patient: case report. J Chemother. 2010;22(1):63-5.

8. Naranjo CA, Busto U, Sellers EM. A method for estimating the probability of adverse drug reactions. Clin Pharmacol Ther. 1981;30(2):239-45.

9. Wang GJ, Gao CF, Wei D, Wang C, Ding SQ. Acute pancreatitis: etiology and common pathogenesis. World J Gastroenterol. 2009;15(12): 1427-30.

10. Hung WY, Lanfranco OA. Contemporary review of drug-induced pancreatitis: a different perspective. World J Gastrointest Pathophysiol. 2014;5(4):405-15.

11. Trivedi CD, Pitchumoni CS. Drug-induced pancreatitis: an update. J Clin Gastroenterol. 2005;39(8):709-16.

12. Badalov N, Baradarian R, Iswara K, Steinberg W, Tenner S. Druginduced pancreatitis: an evidence-based review. Clin Gastroenterol Hepatol. 2007;5(6):648-61.

13. Nakagawa N, Ochi N, Yamane H, Honda Y, Nagasaki Y, Urata N, et al. Ceftriaxone-associated pancreatitis captured on serial computed tomography scans. Radiol Case Rep. 2017;13(1):43-6.

14. Kang SM, Song JE. Ceftriaxone-induced acute pancreatitis in patient with liver abscess. Korean J Pancreas Biliary Tract. 2018;23(2):82-6.
15. Abe S. A case of ceftriaxone-associated biliary pseudolithiasis in an elderly patient with renal dysfunction. IDCases. 2017;9:62-4.

16. Ksiądzyna D. Drug-induced acute pancreatitis related to medications commonly used in gastroenterology. Eur J Intern Med. 2011;22(1):20-5.

Muneerah M Albugami, MD, is with the Department of Medicine, King Faisal Specialist Hospital and Research Centre, Riyadh, Saudi Arabia.

Mohamed Ahmed, MPharm, MRPharmS, BCPS, is with the Pharmaceutical Care Division, King Faisal Specialist Hospital and Research Centre, Riyadh, Saudi Arabia.

Abdulelah Bin Shihah, MD, is with the Department of Family Medicine, King Faisal Specialist Hospital and Research Centre, Riyadh, Saudi Arabia.

Competing interests: None declared.

\section{Address correspondence to:}

Muneerah M Albugami

Department of Medicine

King Faisal Specialist Hospital and Research Centre (KFSHRC)

Riyadh 11211, Saudi Arabia

email: mbugami@kfshrc.edu.sa

Funding: None received.

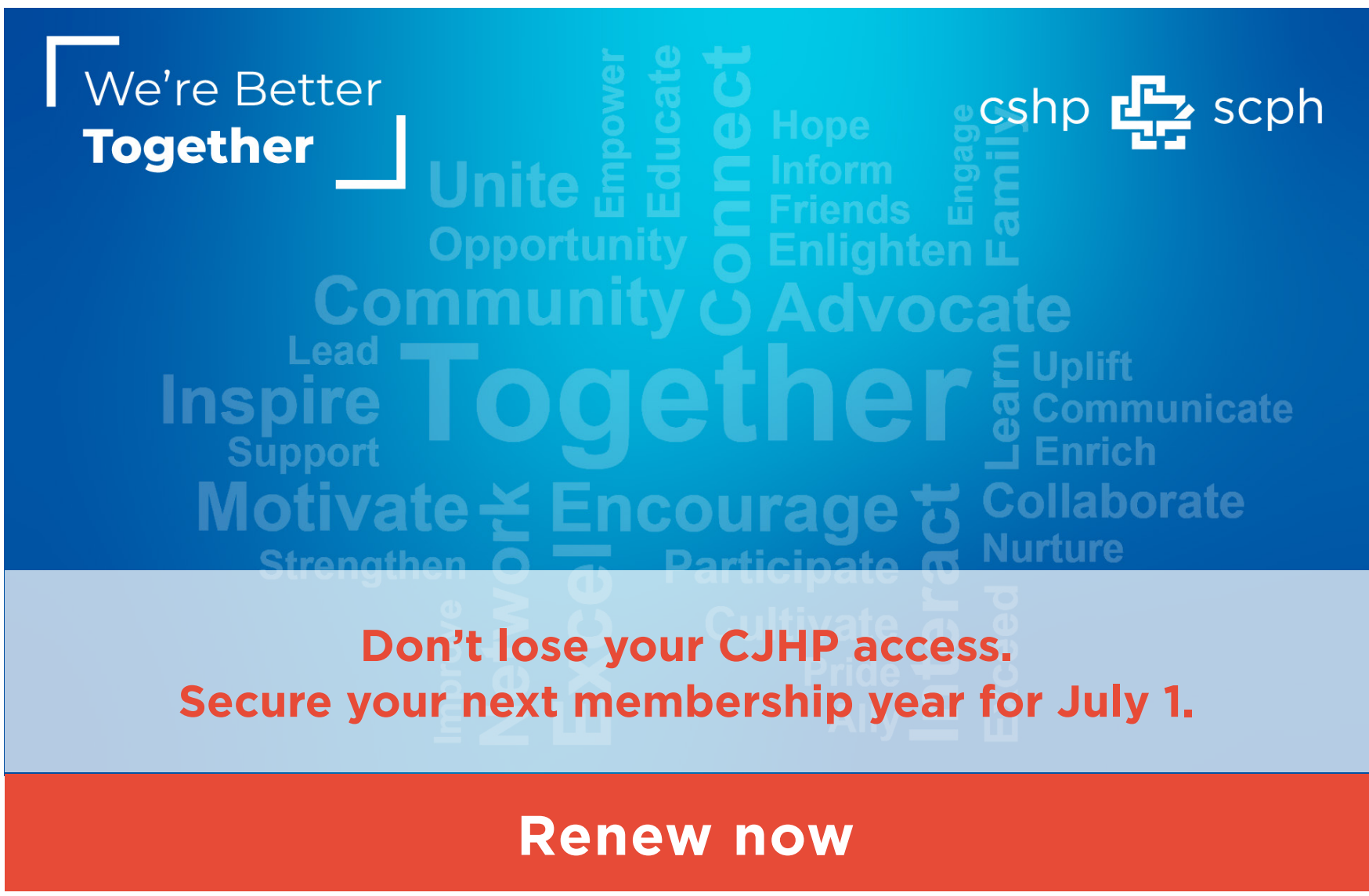

